\title{
Obstructions for Twist Star Products
}

\author{
Pierre Bieliavsky* \\ Faculté des sciences \\ Ecole de mathématique (MATH) \\ Institut de recherche en mathématique et physique (IRMP) \\ Chemin du Cyclotron 2 bte L7.01.02 \\ 1348 Louvain-la-Neuve \\ Belgium \\ Chiara Esposito, Stefan Waldmann ${ }^{\ddagger}$ Thomas Weber ${ }^{\S}$ \\ Institut für Mathematik \\ Lehrstuhl für Mathematik X \\ Universität Würzburg \\ Campus Hubland Nord \\ Emil-Fischer-Straße 31 \\ 97074 Würzburg \\ Germany
}

July 2016

\begin{abstract}
In this short note we point out that not every star product is induced by a Drinfel'd twist by showing that not every Poisson structure is induced by a classical $r$-matrix. Examples include the higher genus symplectic Pretzel surfaces and the symplectic sphere $\mathbb{S}^{2}$.
\end{abstract}

*pierre.bieliavsky@uclouvain.be

${ }^{\dagger}$ chiara.esposito@mathematik. uni-wuerzburg.de

${ }^{\ddagger}$ stefan. waldmann@mathematik. uni-wuerzburg. de

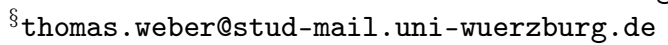




\section{Contents}

1 Introduction $\quad 2$

2 Preliminaries $\quad 3$

3 Drinfel'd twist and transitive actions

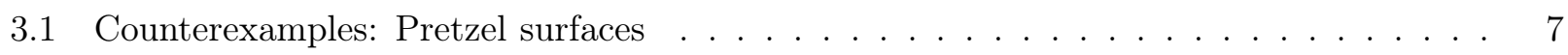

3.2 Counterexample: Symplectic Sphere . . . . . . . . . . . . . . . . 8

\begin{tabular}{ll} 
References & 8 \\
\hline
\end{tabular}

\section{Introduction}

Deformation quantization as introduced in 2] has found many applications in mathematical physics beyond the original scope of quantizing classical mechanical systems. In particular, the formal star products provide examples of noncommutative manifolds as needed in noncommutative geometry [3]. Here one finds many approaches to fundamental questions of the physical nature of the geometry of spacetime.

While for generic star products certain desirable constructions will not be possible, there are particular classes of star products with better behaviour: star products induced by a Drinfel'd twist by means of universal deformation formulas. Recall that for a formal Drinfel'd twist $\mathcal{F} \in(\mathcal{U}(\mathfrak{g}) \otimes \mathfrak{U}(\mathfrak{g}))[[\hbar]]$ one can deform every algebra $\mathscr{A}$ on which the Lie algebra $\mathfrak{g}$ acts by derivations. Indeed, for $a, b \in$ $\mathscr{A}[[\hbar]]$ we can define the product

$$
a \star_{\mathcal{F}} b=m\left(\mathcal{F}^{-1} \triangleright(a \otimes b)\right),
$$

where $m:(\mathscr{A} \otimes \mathscr{A})[[\hbar]] \longrightarrow \mathscr{A}[[\hbar]]$ is the undeformed multiplication of $\mathscr{A}$, extended $\hbar$-bilinearly, and $\triangleright$ denotes the Lie algebra action extended to an action of the universal enveloping algebra as usual. The conditions on $\mathcal{F}$ guarantee that $\star_{\mathcal{F}}$ is again an associative product whenever $m$ was associative.

Beside this simple and universal formula, deformations by means of a twist allow for many additional constructions. In particular, every module over the undeformed algebra on which the Lie algebra acts as well, can be deformed into a module over the deformed algebra. In noncommutative geometry this aspect is of great importance: here we consider as algebra to start with the functions $\mathscr{A}=\mathscr{C}^{\infty}(M)$ on a manifold $M$. Then a Lie algebra action on $\mathscr{A}$ is the same thing as a Lie algebra action by vector fields on $M$. Hence this action lifts to all tensor bundles over $M$. Therefore we can deform the sections of all tensor bundles over $M$ in a coherent way. For a general star product this would not be possible in such a nice way: instead, the sections of every vector bundle have to be deformed individually but with no significant relations between different bundles.

While providing nice additional features, the question has to be raised whether such star products occur rarely or often: one has of course many examples at hand but it is not clear whether there are hard obstructions for a given star product to be induced by a twist. Moreover, recall that a star product induces as semiclassical limit on $M$ a Poisson structure. Thus a natural question is whether a given Poisson structure allows for a star product induced by a twist.

In this work we provide several obstructions for this to be possible. First, we have some quite general obstructions in the case when the Poisson bracket is symplectic:

Theorem 1.1 Given a connected and compact symplectic manifold $(M, \omega)$ endowed with a star product induced by a twist of some Lie algebra. Then $M$ is homogeneous and there exists a non-degenerate $r$-matrix for a (possibly different) Lie algebra whose Lie group acts transitively and effectively on $M$. 
Using this theorem, we show that the general obstructions can be realized in many explicit examples like for the symplectic sphere $\mathbb{S}^{2}$ or all higher genus Pretzel surfaces.

The strategy is to show first that the first order of the twist, the classical $r$-matrix associated to the twist, induces the Poisson structure coming from the star product $\star_{\mathcal{F}}$ : this is the semiclassical version of (1.1). In a second step, one uses the fact that $r$ is contained in a Lie subalgebra in such a way that $r$ becomes non-degenerate there. Third, we show that in the symplectic case the action of this subalgebra is infinitesimally transitive. If it is integrable, say for $M$ compact, and if $M$ is connected, then we obtain a transitive action. Finally, a last argument shows that passing to the quotient by the kernel of the action does not spoil this picture.

Counterexamples are then easily obtained by either symplectic manifolds which are not homogeneous at all, like the higher genus Pretzel surfaces, or by homogeneous manifolds where none of the acting Lie group admits a non-degenerate $r$-matrix: this will be the case for the sphere $\mathbb{S}^{2}$.

As a last remark we note that there is also a slightly weaker concept than star products induced by a twist: we only require that the star product allows for a braiding structure encoded by a universal $R$-matrix $R$. It turns out that also in this case, we arrive at the same semiclassical obstructions and hence the above statements gives also obstructions for such braiding structures.

This paper is partially based on the master thesis [16].

Acknowledgements: We would like to thank Martin Bordemann, Alexander Schenkel and Jonas Schnitzer for valuable discussions and useful suggestions.

\section{Preliminaries}

Let $\mathfrak{g}$ be a finite-dimensional real Lie algebra and consider the algebra $\mathcal{U}(\mathfrak{g})[[\hbar]]$ of formal power series with coefficients in the universal enveloping algebra $\mathcal{U}(\mathfrak{g})$. It is known that it can be easily endowed with a topologically free Hopf algebra structure, denoted by $(\mathcal{U}(\mathfrak{g})[[\hbar]], \Delta, \epsilon, S)$. To fix our notation and sign conventions, we recall the following two definitions of a Drinfel'd twist and a (classical) $r$-matrix, see [4, 5].

Definition 2.1 (Drinfel'd twist) An element $\mathcal{F} \in(\mathcal{U}(\mathfrak{g}) \otimes \mathcal{U}(\mathfrak{g}))[[\hbar]]$ is said to be a twist on $\mathcal{U}(\mathfrak{g})[[\hbar]]$ if the following three conditions are satisfied.

i.) $\mathcal{F}=1 \otimes 1+\sum_{k=1}^{\infty} \hbar^{k} \mathcal{F}_{k}$.

ii.) $(\mathcal{F} \otimes 1)(\Delta \otimes 1)(\mathcal{F})=(1 \otimes \mathcal{F})(1 \otimes \Delta)(\mathcal{F})$.

iii.) $(\epsilon \otimes 1) \mathcal{F}=(1 \otimes \epsilon) \mathcal{F}=1$.

Let $\llbracket \cdot, \cdot \rrbracket$ be the unique extension of the Lie bracket to $\Lambda^{\bullet} \mathfrak{g}$ turning the Grassmann algebra into a Gerstenhaber algebra.

Definition 2.2 ( $r$-Matrix) An element $r \in \mathfrak{g} \wedge \mathfrak{g}$ is said to be a classical $r$-matrix if it satisfies the classical Yang-Baxter equation $\llbracket r, r \rrbracket=0$.

The relation between the two concepts is well-known to be as follows, see [5] or [7, Thm. 1.14]:

Proposition 2.3 Let $\mathcal{F}$ be a twist on $\mathcal{U}(\mathfrak{g})[[\hbar]]$. Then the antisymmetric part of its first order

$$
r=\mathcal{F}_{1}^{-1}-\sigma\left(\mathcal{F}_{1}^{-1}\right)=-\mathcal{F}_{1}+\sigma\left(\mathcal{F}_{1}\right) \in \mathcal{U}(\mathfrak{g}) \wedge \mathcal{U}(\mathfrak{g})
$$

is a classical $r$-matrix $r \in \mathfrak{g} \wedge \mathfrak{g}$. Here $\sigma$ denotes the flip isomorphism.

It is important to recall that there is a one-to-one correspondence between non-degenerate $r$ matrices and symplectic structures on $\mathfrak{g}$, see [6, Prop. 3.3]. For this reason, we are interested to discuss, given a Lie algebra $\mathfrak{g}$, under which conditions it is possible to endow it with a non-degenerate $r$-matrix. It turns out that if $\mathfrak{g}$ is semisimple, this is not possible, see [6. Prop. 5.2] for the complex and simple case, which clearly extends to real and semisimple Lie algebras: 
Proposition 2.4 Let $\mathfrak{g}$ be a semisimple Lie algebra. Then there are no non-degenerate $r$-matrices on it.

On the other hand, one can always find a suitable (finite-dimensional) Lie subalgebra $\mathfrak{g}_{r} \subseteq \mathfrak{g}$ such that an $r$-matrix on $\mathfrak{g}$ is non-degenerate if viewed as an element of $\mathfrak{g}_{r} \wedge \mathfrak{g}_{r}$, see [6. Sect. 3.5]:

Proposition 2.5 Let $r \in \mathfrak{g} \wedge \mathfrak{g}$ be an r-matrix for a real Lie algebra $\mathfrak{g}$.

i.) The subspace $\mathfrak{g}_{r}$ defined by

$$
\mathfrak{g}_{r}=\left\{(f \otimes \mathbb{1}) r \in \mathfrak{g} \mid f \in \mathfrak{g}^{*}\right\}
$$

is a finite-dimensional Lie subalgebra of $\mathfrak{g}$.

ii.) We have $r \in \mathfrak{g}_{r} \wedge \mathfrak{g}_{r}$.

iii.) $r$ is non-degenerate, if viewed as en element $r \in \mathfrak{g}_{r} \wedge \mathfrak{g}_{r}$.

Definition 2.6 (Symplectic subalgebra) The Lie subalgebra $\mathfrak{g}_{r} \subseteq \mathfrak{g}$ defined in (2.2) is called the symplectic subalgebra of the $r$-matrix $r \in \mathfrak{g} \wedge \mathfrak{g}$.

In the case of a real Lie algebra $\mathfrak{g}$, the corresponding Lie group is denoted by $G_{r}$.

As already mentioned, given a twist on the universal enveloping algebra, we can easily define an associative star product on any $\mathcal{U}(\mathfrak{g})$-module algebra. In particular, let us consider the algebra $\mathscr{C}^{\infty}(M)$ of smooth functions on a manifold $M$ with pointwise multiplication $m$ and assume that it is a left $\mathcal{U}(\mathfrak{g})$-module algebra. In other words, let us consider a Hopf algebra action

$$
\triangleright: \mathcal{U}(\mathfrak{g}) \times \mathscr{C}^{\infty}(M) \longrightarrow \mathscr{C}^{\infty}(M),
$$

which makes $\mathscr{C}^{\infty}(M)$ into a left $\mathcal{U}(\mathfrak{g})$-module algebra. Since $\mathfrak{g} \subseteq \mathcal{U}(\mathfrak{g})$ are the primitive elements, the Lie algebra elements act as derivations of $\mathscr{C}^{\infty}(M)$, i.e. as vector fields on $M$. This defines a Lie algebra action $\phi$ of $\mathfrak{g}$ on $M$. Since the elements of $\mathfrak{g}$ generate $\mathcal{U}(\mathfrak{g})$, the action $\triangleright$ is given by differential operators: more precisely, using the natural filtration of the universal enveloping algebra, the order of the differential operator $X \triangleright$. is at most $k \in \mathbb{N}$ when $X \in \mathcal{U}^{(k)}(\mathfrak{g})$. From now on we also denote by $\triangleright$ the extension of the action to formal power series $\triangleright: \mathcal{U}(\mathfrak{g})[[\hbar]] \times \mathscr{C}^{\infty}(M)[[\hbar]] \longrightarrow \mathscr{C}^{\infty}(M)[[\hbar]]$. Conversely, every Lie algebra action $\phi$ of $\mathfrak{g}$ on $M$ determines via the fundamental vector fields $\phi(\xi) \in \Gamma^{\infty}(T M)$ a representation of $\mathfrak{g}$ on $\mathscr{C}^{\infty}(M)$ by derivations which therefore extends to a Hopf algebra action $\triangleright$ as above.

Since the first-order commutator of an associative commutative algebra is necessarily a Poisson bracket on the (un-deformed) algebra, see e.g. [15, Proposition 6.2.24], we have the following statement:

Lemma 2.7 The product defined by

$$
f \star \mathcal{F} g=m\left(\mathcal{F}^{-1} \triangleright(f \otimes g)\right)
$$

for $f, g \in \mathscr{C}^{\infty}(M)[[\hbar]]$ is an associative star product quantizing the Poisson structure

$$
\{f, g\}=m(r \triangleright(f \otimes g)),
$$

where $r$ is the $r$-matrix associated to the twist $\mathcal{F}$.

Remark 2.8 (Taking the classical limit) We can summarize this discussion now as follows. Taking the classical limit of a twist $\mathcal{F}$ gives a classical $r$-matrix $r$. Taking the classical limit of a Hopf algebra action $\triangleright$ of $\mathcal{U}(\mathfrak{g})[[\hbar]]$ and restricting it to the Lie algebra gives a Lie algebra action $\phi$ on $M$ by vector fields, and taking the classical limit of a star product gives a Poisson bracket. If now the star product comes from a twist via some Hopf algebra action then the corresponding Poisson bracket comes from the corresponding $r$-matrix and the corresponding Lie algebra action. 
Finally, we will also need the quantum analogue of $r$-matrices:

Definition 2.9 (Universal $R$-matrix) An invertible element $R \in(\mathcal{U}(\mathfrak{g}) \otimes \mathcal{U}(\mathfrak{g}))[[\hbar]]$ is called a formal universal $R$-matrix if

i.) $R=1 \otimes 1+\cdots$,

ii.) $\Delta^{\mathrm{opp}}=R \Delta R^{-1}$,

iii.) $(1 \otimes \Delta) R=\left(R_{1} \otimes 1 \otimes R_{2}\right)(R \otimes 1)$ and $(\Delta \otimes 1) R=\left(R_{1} \otimes 1 \otimes R_{2}\right)(1 \otimes R)$, using Sweedler's notation $R=R_{1} \otimes R_{2}$.

It is known that $R$ is the quantization of the $r$-matrix over $\mathfrak{g}$. In other words, given a formal $R$-matrix $R$ for $\mathfrak{g}$, we can define an $r$-matrix for $\mathfrak{g}$ by the first order term

$$
R=1 \otimes 1+\hbar r+\cdots .
$$

A star product $\star$ is called quasi-commutative with respect to $R$ if one has an action of $\mathfrak{g}$ by derivations such that the extended actions $\triangleright$ satisfies

$$
f \star g=\left(R_{2} \triangleright g\right) \star\left(R_{1} \triangleright f\right)
$$

for all $f, g \in \mathscr{C}^{\infty}(M)[[\hbar]]$, see [1, Def. 5.8]. A simple verification leads to the following statement:

Proposition 2.10 Suppose $\star$ is a quasi-commutative star product on $M$ with respect to a universal $R$-matrix $R$ for $\mathfrak{g}$. Then the corresponding Poisson structure of $\star$ is induced by the corresponding $r$-matrix $r$.

\section{Drinfel'd twist and transitive actions}

In this section we prove the main result of this paper, which allows us to understand the conditions under which a star product on a manifold $M$ can be induced by a twist on the universal enveloping algebra of a Lie algebra $\mathfrak{g}$ acting on $M$.

Let $r$ be an $r$-matrix on $\mathfrak{g}$. For a finite-dimensional Lie algebra $\mathfrak{g}$ with basis $\left\{e_{1}, \ldots, e_{n}\right\}$, we have

$$
r=\frac{1}{2} \sum_{i, j=1}^{n} r^{i j} e_{i} \wedge e_{j}
$$

Given a Lie algebra action $\phi: \mathfrak{g} \rightarrow \Gamma^{\infty}(T M)$ we can define a Poisson structure on $M$ as the image via $\phi$ of the $r$-matrix, i.e.

$$
\pi_{p}=\frac{1}{2} \sum_{i, j=1}^{n} r^{i j} \phi\left(e_{i}\right)_{p} \wedge \phi\left(e_{j}\right)_{p},
$$

for $p \in M$. If $\pi$ is non-degenerate, it induces a symplectic structure via $\omega\left(X_{f}, X_{g}\right)=\pi(\mathrm{d} f$, $\mathrm{d} g)$, where $X_{f}$ denotes the Hamiltonian vector field of $f \in \mathscr{C}^{\infty}(M)$ with respect to $\pi$.

In order to prove our main result we need some preparation. First we have to pass from a Lie algebra action to a Lie group action of an integrating Lie group $G$ of $\mathfrak{g}$. For the moment we have to assume that this is the case.

Lemma 3.1 Let $(M, \omega)$ be a symplectic manifold, $r \in \mathfrak{g} \wedge \mathfrak{g}$ an $r$-matrix and let $\phi$ be a Lie algebra action of $\mathfrak{g}$ on $M$ integrating to a Lie group action $\Phi: G \times M \rightarrow M$ such that $\omega$ is the image of $r$ via (3.2). Then $\Phi$ is locally transitive. Moreover, the restriction $\left.\Phi\right|_{G_{r}}$ of $\Phi$ to the Lie subgroup $G_{r}$ corresponding to $r$ is locally transitive. 
ProOF: It suffices to show the second statement. We observe that we can find a basis $\left\{e_{1}, \ldots, e_{n}\right\}$ of $\mathfrak{g}$ such that there is an integer $k \leq n$ such that $\left\{e_{1}, \ldots, e_{k}\right\}$ is a basis of the symplectic Lie subalgebra $\mathfrak{g}_{r}$ determined by $r$ and

$$
r=\frac{1}{2} \sum_{i=1}^{k} r^{i j} e_{i} \wedge e_{j}
$$

Now consider an arbitrary vector $v_{p} \in T_{p} M$, for $p \in M$. Since for a symplectic Poisson tensor, the map $\pi^{\sharp}: T_{p}^{*} M \ni \alpha_{p} \mapsto \pi_{p}\left(\cdot, \alpha_{p}\right) \in T_{p} M$ is surjective, there exists an element $\alpha_{p} \in T_{p}^{*} M$ such that

$$
v_{p}=\pi_{p}\left(\alpha_{p}, \cdot\right)=\left(\alpha_{p} \otimes \mathbb{1}\right) \pi_{p}=\sum_{i, j=1}^{n} r^{i j} \alpha_{p}\left(\phi\left(e_{i}\right)_{p}\right) \phi\left(e_{j}\right)_{p}=\sum_{i, j=1}^{k} r^{i j} \alpha_{p}\left(\phi\left(e_{i}\right)_{p}\right) \phi\left(e_{j}\right)_{p} .
$$

In other words,

$$
v_{p} \in \operatorname{span}_{\mathbb{R}}\left\{\sum_{j=1}^{k} r^{1 j} \phi\left(e_{j}\right)_{p}, \ldots, \sum_{j=1}^{k} r^{k j} \phi\left(e_{j}\right)_{p}\right\} \subseteq \operatorname{span}_{\mathbb{k}}\left\{\phi\left(e_{1}\right)_{p}, \ldots, \phi\left(e_{k}\right)_{p}\right\} .
$$

Thus, the map $\left.\phi\right|_{p}: \mathfrak{g}_{r} \longrightarrow T_{p} M$ is surjective and hence the action of $G_{r}$ is locally transitive.

A locally transitive action is known to be transitive if the underlying manifold $M$ is connected, and hence a homogeneous space:

Lemma 3.2 Let $\Phi: G \times M \longrightarrow M$ be a locally transitive Lie group action on a connected manifold $M$. Then there is only one orbit of $\Phi$ and it coincides with $M$.

Putting these results together, we can prove the main theorem of this paper:

Theorem 3.3 Let $(M, \omega)$ be a connected symplectic manifold, $r \in \mathfrak{g} \wedge \mathfrak{g}$ an $r$-matrix and $\phi$ a Lie algebra action of $\mathfrak{g}$ on $M$ that integrates to a Lie group action $\Phi: G \times M \longrightarrow M$ such that, for any $p \in M$, the symplectic bivector field $\pi$ corresponding to $\omega$ is given by (3.2). Then $M$ can be structured as a homogeneous space for the Lie subgroup $G_{r}$ corresponding to the symplectic Lie algebra $\mathfrak{g}_{r}$ with respect to $r$.

Proof: This is a direct consequence of Lemma 3.1 and Lemma 3.2.

The first important consequence of this statement is that under the assumption that the Lie algebra action integrates, we have an obstruction for star products to be induced by twists. As before, we denote by $\mathfrak{g}_{r}$ the symplectic Lie subalgebra with respect to the $r$-matrix $r$ corresponding to the twist $\mathcal{F}$ and $G_{r}$ is the connected and simply-connected Lie group integrating it.

Corollary 3.4 Let $(M, \omega)$ be a connected symplectic manifold. Assume that there is a star product $\star_{\mathcal{F}}$ induced by a twist via the action $\triangleright$ such that the corresponding Lie algebra action integrates to a Lie group action. Then $M$ is a homogeneous $G_{r}$-space.

Corollary 3.5 Let $(M, \omega)$ be a connected and compact symplectic manifold with a star product $\star \mathcal{F}$ induced by a twist. Then $M$ is a homogeneous $G_{r}$-space.

PROOF: If we assume compactness then any Lie algebra action comes from a Lie group action by Palais' theorem [14] since the fundamental vector fields are necessarily complete.

We can even say something more about the homogeneous space. There is of course a certain redundancy in the Lie group at the moment: the action could have a large kernel. The following statement shows that we can pass from a transitive action to a transitive and effective action without loosing the $r$-matrix: 
Proposition 3.6 Let $\Phi: G \times M \longrightarrow M$ be a smooth Lie group action on a manifold $M$ with kernel

$$
\operatorname{ker} \Phi=\left\{g \in G \mid \Phi_{g}=\mathrm{id}_{M}\right\}
$$

and let $\operatorname{ker} \phi \subseteq \mathfrak{g}$ be the corresponding Lie ideal.

i.) If the action $\Phi$ is transitive, the induced action

$$
\Psi: G / \operatorname{ker} \Phi \times M \ni([g], x) \mapsto \Phi(g, x) \in M
$$

of the quotient Lie group $G / \operatorname{ker} \Phi$ on $M$ is effective and still transitive.

ii.) For a classical $r$-matrix $r \in \Lambda^{2} \mathfrak{g}$ the image $[r] \in \Lambda^{2} \mathfrak{g} / \operatorname{ker} \phi$ is a classical $r$-matrix for $\mathfrak{g} / \operatorname{ker} \phi$.

iii.) The induced Poisson structures of $r$ and $[r]$ on $M$ coincide.

Proof: The first part is clear and so is the second. Since the Poisson structure on $M$ is obtained by applying $\phi \wedge \phi$ to $r$, the contributions in the kernel ker $\phi$ will not contribute and hence $[r]$ yields the same Poisson structure.

Corollary 3.7 Let $(M, \omega)$ be a compact symplectic manifold such that the Poisson structure is induced by an $r$-matrix. Then there is a non-degenerate $r$-matrix $r \in \Lambda^{2} \mathfrak{g}$ in a Lie algebra $\mathfrak{g}$ with corresponding Lie group $G$ acting transitively and effectively on $M$.

Proof: By Theorem 3.3 we can assume that there is an $r$-matrix $r \in \Lambda^{2} \tilde{\mathfrak{g}}$ in some Lie algebra $\tilde{\mathfrak{g}}$ such that the corresponding group $\tilde{G}$ acts transitively on $M$ via $\Phi$. Then we can quotient by the kernel of the action $\Phi$ to obtain a transitive and effective action of $G=\tilde{G} / \operatorname{ker} \Phi$, still having a classical $r$-matrix inducing the same Poisson bracket. Finally, we can pass to the symplectic Lie subalgebra $\mathfrak{g}_{r}$ and take the Lie subgroup $G_{r}$ of $G$ which still acts transitively and effectively.

\subsection{Counterexamples: Pretzel surfaces}

The above results provide a large class of examples in which star products can not be induced via a twist. The first class of examples that we discuss here is given by manifolds which can not be written as homogeneous spaces at all. For this reason, it is important to recall some useful characterization of homogeneous spaces in terms of Euler characteristic $\chi(M)$. The following classical theorem of Mostow gives now a necessary condition for a manifold to be homogeneous [10], see [9] for the case of surfaces:

Theorem 3.8 (Mostow) Let $M=G / H$ be a connected compact homogeneous space. Then $\chi(M) \geq$ 0 .

From this we immediately get the following class of examples of symplectic manifolds which do not allow a star product induced by a twist:

Example 3.9 (Pretzels) Let us consider the Pretzel surfaces $T(g)$ of genus $g \in \mathbb{N}_{0}$, which are compact and connected symplectic manifolds with Euler characteristic

$$
\chi(T(g))=2-2 g .
$$

For $g>1$ the Euler characteristic is negative. Thus, according to Theorem 3.8 the Pretzel surfaces $T(g)$ are not homogeneous spaces for $g>1$ at all. As an immediate consequence of Corollary [3.5. no symplectic star product on $T(g)$ for $g>1$ can be induced by a twist.

By the same line of argument, one can of course also construct examples of compact symplectic manifolds in higher dimensions. 


\subsection{Counterexample: Symplectic Sphere}

While Mostow's Theorem provides an easy way to rule out the higher genus Pretzel surfaces, the situation for the symplectic sphere $\mathbb{S}^{2}$ and the symplectic torus $\mathbb{T}^{2}$ is more involved: for the torus one can use e.g. the canonical action of $\mathbb{T}^{2}$ on itself. Then the abelian twist provides a star product, the Weyl-Moyal star product, on the torus. So here we do not have any obstructions.

Also the symplectic sphere is a very well-known example of a homogeneous space. Nevertheless, we can show that also in this case no star product on the symplectic sphere can be induced by a twist. The main idea is that even though the sphere is a homogeneous space in several ways, none of them allows for a twist: one uses the well-known classification of transitive and effective actions on $\mathbb{S}^{2}$ and shows by hand that all of them come from semisimple Lie groups. The classification results we use are due to Onishchik [11 13] as well as earlier work by Montgomery and Samelson [8]:

Theorem 3.10 (Onishchik) Any connected Lie group that acts transitively and locally effectively on $\mathbb{S}^{2}$ is semisimple.

Using this classification result together with Corollary 3.7 and Proposition 2.4 we arrive at the following statement for the symplectic sphere:

Proposition 3.11 A symplectic Poisson structure on $\mathbb{S}^{2}$ can not be induced by a classical $r$-matrix.

Corollary 3.12 There is no star product on the symplectic sphere $\mathbb{S}^{2}$ induced by a twist.

\section{References}

[1] Aschieri, P., Schenkel, A.: Noncommutative connections on bimodules and Drinfel'd twist deformation. Adv. Theor. Math. Phys. 18.3 (2014), 513-612.

[2] Bayen, F., Flato, M., Frønsdal, C., Lichnerowicz, A., Sternheimer, D.: Deformation Theory and Quantization. Ann. Phys. 111 (1978), 61-151.

[3] Connes, A.: Noncommutative Geometry. Academic Press, San Diego, New York, London, 1994.

[4] Drinfel'D, V. G.: On constant quasiclassical solutions of the Yang-Baxter quantum equation. Sov. Math. Dokl. 28 (1983), 667-671.

[5] Drinfeld, V. G.: Quantum groups. J. Sov. Math. 41 (1988), 898-918.

[6] Etingof, P., Schiffmann, O.: Lectures on Quantum Groups. International Press, Boston, 1998.

[7] Giaquinto, A., Zhang, J. J.: Bialgebra actions, twists, and universal deformation formulas. J. Pure Appl. Algebra 128.2 (1998), 133-152.

[8] Montgomery, D., Samelson, H.: Transformation Groups of Spheres. Annals of Mathematics, Vol. 44, No. 3 (Jul., 1943), 1942.

[9] Mostow, G. D.: The extensibility of local Lie groups of transformations and groups on surfaces. Ann. of Math. (2) $52(1950), 606-636$.

[10] Mostow, G. D.: A structure theorem for homogeneous spaces. Geom. Dedicata 114 (2005), 87-102.

[11] Onishchik, A.: On Lie Groups Transitive on Compact Manifolds II. Mathematics of the USSR-Sbornik 3.3 (1967), 373 .

[12] Onishchik, A.: On Lie Groups Transitive on Compact Manifolds III. Mathematics of the USSR-Sbornik 4.2 (1968), 233.

[13] Onishchik, A.: Lie Groups and Lie Algebras I. Encyclopaedia of mathematical sciences. Springer-Verlag, 1993.

[14] Palais, R. S.: A global formulation of the Lie theory of transformation groups, vol. 22. 1957.

[15] Waldmann, S.: Poisson-Geometrie und Deformationsquantisierung. Eine Einführung. Springer-Verlag, Heidelberg, Berlin, New York, 2007.

[16] Weber, T.: Star Products that can not be induced by Drinfel'd Twists. master thesis, University of Würzburg, Würzburg, Germany, 2016. 